\title{
SUSTENTABILIDADE DOS AGROECOSSISTEMAS INTENSIVOS DE BANANEIRA DE IPANGUAÇU-RN
}

\author{
L. M. M. Reis ${ }^{1,2}$, G. A. Cândido² \\ ${ }^{1}$ Instituto Federal de Educação Ciência e Tecnologia do Rio Grande do Norte \\ ${ }^{2}$ PPG em Recursos Naturais - Universidade Federal de Campina Grande \\ leci.reis@ifrn.edu.br, gacandido@uol.com.br
}

\begin{abstract}
Artigo submetido em setembro/2012 e aceito em janeiro/2013
\section{RESUMO}

O artigo aborda a sustentabilidade dos agroecossistemas intensivos de bananeira situados no município de Ipanguaçu, estado do Rio Grande do Norte, que fazem uso de alta tecnologia. Esse tipo de sistema agrícola tem sido questionado, por ter-se voltado para os princípios da revolução verde, de modo que, no curso dos últimos tempos, têm surgido dúvidas sobre os níveis de sustentabilidade desses agroecossistemas. Com a construção da Barragem Armando Ribeiro Gonçalves, no município de Ipanguaçu-RN, com a estruturação do polo fruticultor, impulsionado, dentre outras razões, pelo incremento tecnológico hídrico instalado nessa área, que outrora sofria com escassez de água. Objetiva avaliar a sustentabilidade desses agroecossistemas

intensivos de bananeira no ano de 2011. A metodologia do estudo consta de pesquisa bibliográfica, documental, campo (GIL, 2009) e estudo de caso (YIN, 2007), seguindo-se o método biograma (SEPÚLVEDA, 2008). Para aplicar o método foram consideradas as dimensões ambiental, econômica e social, com a definição de 15 indicadores. Como resultado, os agroecossistemas 2 e 4 apresentaram índice de sustentabilidade de 0,33 e de 0,24, indicando um estado crítico e um estado instável, respectivamente; o agroecossistema 1 obteve o índice de 0,47, o que indica um estado instável; por fim, o agroecossistema 3 atingiu o índice de 0,94, demonstrando um estado ótimo e, portanto, apresentando-se como o mais sustentável.
\end{abstract}

Palavras-chave: Sustentabilidade. Agricultura sustentável. Agroecossistemas intensivos.

\section{SUSTENTABILIDADE DOS AGROECOSSISTEMAS INTENSIVOS DE BANANEIRA DE IPANGUAÇU-RN}

\section{ABSTRACT}

The article discusses the sustainability of agroecosystems intensive banana situated in the municipality of Ipanguaçu, state of Rio Grande do Norte, which make use of high technology. This type of farming system has been questioned, having turned to the principles of green revolution, so that in the course of recent times, there have been doubts about the sustainability of agroecosystems levels. With the construction of the Dam Armando Ribeiro Gonçalves, in the municipality of Ipanguaçu-RN, with the structuring polo grower, driven, among other reasons, by increasing water technology installed in this area, which once suffered from water shortages. Aims to evaluate the sustainability of agroecosystems intensive banana in 2011. The study methodology included a literature review, documentary field (GIL, 2009) and case study (Yin, 2007), following the method Biogram (SEPÚLVEDA, 2008). To apply the method were considered the environmental, economic and social, with the definition of 15 indicators. As a result, 2 and 4 showed agroecosystems sustainability index of 0.33 and 0.24 , indicating a critical state and an unstable state, respectively, the agroecosystem 1 obtained the index of 0.47 , which indicates an unstable state and finally, the agroecosystem 3 has reached the level of 0.94 , demonstrating an optimal state and therefore presenting itself as the most sustainable.

KEY-WORDS: Sustainability. Sustainable agriculture. Intensive agroecosystems. 


\section{INTRODUÇÃO}

Este artigo aborda a sustentabilidade de agroecossistemas intensivos de bananeira situados no município de Ipanguaçu, no estado do Rio Grande do Norte (Figura 1), classificados como estabelecimentos rurais que utilizam alta tecnologia ${ }^{1}$. Esse tipo de sistema agrícola tem sido amplamente questionado devido a sua dinâmica ter-se voltado basicamente para os princípios da revolução verde. Talvez seja por isso que, no curso dos últimos tempos, têm surgido dúvidas sobre a produtividade, em longo prazo, da fruticultura intensiva, baseada nos insumos externos, e sobre a sustentabilidade desse tipo de agricultura (TISDELL, 1999).

Figura 1: Localização do município de Ipanguaçu-RN

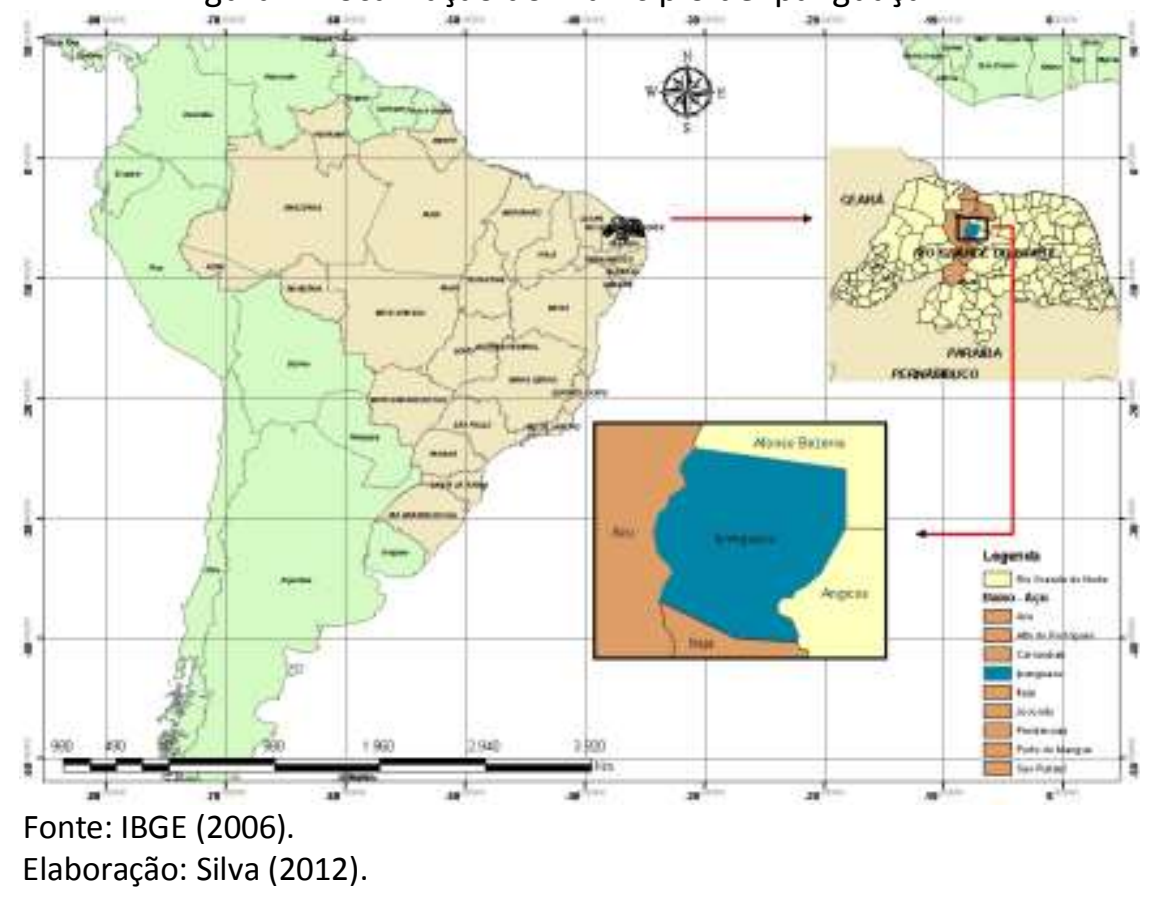

Com se sabe, a revolução verde tem se caracterizado por seu avanço científico e tecnológico, proporcionando a produção e o uso de agroquímicos, a maximização produtiva e o lucro, mas também impactos, traduzidos em efeito estufa, desmatamento, chuvas ácidas, dentre outros, que têm ameaçado a sobrevivência e a qualidade da vida humana. Esse tipo de agricultura tem sido elogiado por uns, devido à grande produção de alimentos á geração de riqueza (BORLAUG, 1997; 2008), e apontado por outros como responsável pelos ciclos de degradação e pobreza observados em grandes regiões do planeta (ALTIERI, 2002).

Entretanto, não se desconsidera que a atividade agrícola intensiva tem se voltado basicamente para a produção de alimentos, fibras e energia, visando atender, sobretudo, o aumento populacional e, por conseguinte, o crescimento do consumo e a sobrevivência humana. Estudos como os de Borlaug (1997), abordando a reprodução vegetal, têm permitido o aumento da produção agrícola em diversas regiões. Somando-se a isso, pesquisas sobre a disseminação de variedades de cereais de alto rendimento podem ter contribuído para evitar o aumento da fome causado pela baixa disponibilidade de alimentos de origem agrícola. Os agroecossistemas (termo abreviado neste artigo como AGROs) intensivos, ou irrigados, têm

\footnotetext{
${ }^{1}$ Utiliza insumos externos (agrotóxicos, herbicidas, maquinários, sementes selecionadas, conhecimento técnico cientifico, dentre outros). Nesse processo podem ser percebidos níveis, distintos quanto ao uso de tecnologias e manejo nos AGROs, formando subsistemas, o que emprega: baixa e alta tecnologia, tendo como base inovação científica e tecnológica, modernização da agricultura (DINIZ, 1986, p. 218).
} 
recebido diversas adjetivações. Fala-se em agricultura moderna, intensiva, comercial, convencional, tecnológica, científica, plantation, dentre outras denominações (BORLAUG, 1997; SANTOS; SILVEIRA, 2002).

Juntamente com essas denominações, surgiram as discussões sobre sustentabilidade, que trazem para o debate a importância da inclusão das dimensões ambiental, econômica e social, tendo como eixo norteador as práticas e os manejos ${ }^{2}$ dos recursos naturais que podem delinear sistemas agrícolas sustentáveis (SACHS, 2004). Esses sistemas levam em consideração à relação do homem-natureza, isto é, a interação entre os aspectos sociais e os recursos naturais dos sistemas agrícolas, definidos como o conjunto das atividades realizadas no local, considerando-se os elementos biofísicos e os socioeconômicos (SCHULTZ, 1965).

Algumas dessas práticas são vivenciadas, empiricamente, nos agroecossistemas intensivos de Ipanguaçu-RN e, teoricamente, em estudos como o de Sampaio (2003), que discute a participação do agronegócio na economia brasileira; o de Gomes (1999), que aborda a dinamização da fruticultura no Vale do Açu; o de Albano (2008), que trata da globalização da agricultura e da concentração fundiária no município de Ipanguaçu-RN; o de Antas (2011), que avalia o índice da qualidade da água para irrigação do Rio Piranhas-Açu; dentre outros, que têm contribuído para a discussão da problemática em foco.

Com a construção da Barragem Armando Ribeiro Gonçalves, mais conhecida como Barragem do Açu, surgiu um novo sistema produtivo no vale do rio Piranhas-Açu, o que contribuiu para a estruturação do polo fruticultor, impulsionado, dentre outras razões, pelo incremento tecnológico hídrico instalado na região, a qual outrora sofriam com a escassez de água. No sistema produtivo de Ipanguaçu-RN, ocorreram transformações, surgindo novas formas de produção, as quais promoveram um novo sistema agrícola, do tipo intensivo, de fruticultura, diferente do que historicamente era desenvolvido pelo complexo agropecuário: agricultura de subsistência (feijão, milho e batata-doce), cultivo do algodão, criação de gado ${ }^{3}$ e extração da cera de carnaúba. Com esse fenômeno, emerge uma situação conflituosa, para muitos, questionando-se se os agroecossistemas intensivos de bananeira, que se configuram como agentes transformadores do perfil agrícola local, são sustentáveis.

Diante dessa incerteza, este estudo tem como objetivo avaliar, comparativamente, a sustentabilidade de agroecossistemas intensivos de bananeira, de alta tecnologia, de Ipanguaçu, Rio Grande do Norte, Brasil, no ano agrícola de 2011, sob a ótica do biograma. A metodologia adotada caracteriza-se como pesquisa bibliográfica, documental, campo (GIL, 2009) e estudo de caso (YIN, 2007), seguindo-se o método do desenvolvimento integrado sustentável (IDS) - biograma -, poposto por Sepúlveda, (2008), o qual se destina à avaliação da sustentabilidade do território rural, ou unidade de análise, e tem caráter multidimensional dimensões ambiental, econômica, social e político-institucional -, considerando-se determinado espaço-tempo sob os vieses da sustentabilidade, da equidade e da produtividade (HAMMOND et al., 1995).

Nos agroecossistemas intensivos de Ipanguaçu-RN, o cultivo da bananeira tem tido certa hegemonia quanto à dimensão das áreas plantadas (Tabela 1) e à produtividade (Tabela 2).

\footnotetext{
${ }^{2}$ Execução de procedimentos que interferem nas condições ambientais de determinada área, visando incrementar produtividade, melhorar qualidade e agregar valores à matéria-prima, isto é, utilizar os recursos naturais racionalmente, permitindo a continuidade da produção, da rentabilidade, da segurança, da legalidade, do mercado, da preservação dos recursos naturais e dos serviços ambientais (BRASIL, 2000).

${ }^{3}$ Desenvolvida no entorno da Lagoa Ponta Grande (VALVERDE; MESQUITA, 1961; ANDRADE, 1995).
} 
Neles, o novo processo de produção tem se voltado para ações dinamizadoras, como: redução do custo unitário de produção, utilização do solo por meio de manejo adequado, padronização da qualidade dos produtos agrícolas, promoção de segurança alimentar, empregabilidade, redução do fator de risco de produção relacionado à escassez de água, competitividade interna e externa, dentre outras (IBGE, 2011; FIERN, 2011). Com o intuito de mostrar a evolução da ocorrência de áreas plantadas de fruticultura permanente - bananeira, mangueira e goiabeira - no município de Ipanguaçu, apresenta-se, na Tabela 1 a área plantada, por hectare (ha.), no período de 1990 a 2011. No início, predominou áreas plantadas de mangueira, até 1996; a partir de então, essas áreas vêm sendo superadas pelas de bananeira. Enquanto isso, o cultivo da goiaba tem sido inexpressivo.

Tabela 1 - Área plantada de fruticultura permanente de Ipanguaçu-RN

\begin{tabular}{|cccccccc}
\hline & \multicolumn{3}{c}{ Permanente (ha.) } & \multicolumn{3}{c}{ Permanente (ha.) } \\
\hline Ano & Banana & Manga & Goiaba & Ano & Banana & Manga & Goiaba \\
\hline 1990 & 185 & 340 & $-*$ & 2001 & 560 & 500 & 15 \\
\hline 1991 & 185 & 340 & - & 2002 & 1086 & 500 & 15 \\
\hline 1992 & 185 & 340 & - & 2003 & 1086 & 500 & 15 \\
\hline 1993 & 140 & 340 & - & 2004 & 1248 & 500 & 15 \\
\hline 1994 & 140 & 380 & - & 2005 & 1293 & 480 & 10 \\
\hline 1995 & 140 & 340 & - & 2006 & 1273 & 506 & 5 \\
\hline 1996 & 100 & 340 & 16 & 2007 & 1330 & 500 & 5 \\
\hline 1997 & 400 & 340 & 15 & 2008 & 783 & 500 & 5 \\
\hline 1998 & 400 & 500 & 15 & 2009 & 757 & 500 & 5 \\
\hline 1999 & 560 & 500 & 15 & 2010 & 786 & 200 & 5 \\
\hline 2000 & 560 & 500 & 15 & 2011 & 786 & 350 & 5 \\
\hline
\end{tabular}

Fonte: IBGE (2011).

Nota: *Sem informação sobre esse ano agrícola.

Nessa tabela, observa-se que, embora, no curso dos últimos anos, o cultivo da bananeira tenha tido notoriedade no que diz respeito à área de produção, em relação ao das demais frutas há certa redução do número de hectares plantados nos anos de 2008, 2009 e 2010, em relação ao ano de 2007. Essa redução, tem sido atribuída ao excesso de chuvas precipitadas nos anos agrícolas de 2008 e 2009, no semiárido potiguar - Ipanguaçu-RN -, dificultando a realização adequada do manejo de produção e colheita dos agroecossistemas de bananeira. Apesar dessa intempérie pluvial, muitos agroecossistemas intensivos de bananeira já apresentaram certa recuperação no ano de 2010. Tendo em vista os problemas elencados, partimos para a seguinte indagação: quais, dentre os agroecossistemas intensivos de bananeira de alta tecnologia situados em Ipanguaçu-RN, podem ser considerados mais sustentáveis, utilizandose o método biograma?

\section{FUNDAMENTAÇÃO TEÓRICA}

Com o intuído de avaliar a sustentabilidade dos agroecossistemas intensivos, traz-se à tona uma discussão conceitual sobre sustentabilidade. A palavra sustentabilidade tem sua origem no latim sustentare, que significa suportar, ou seja, no caso de nosso estudo, manter em situação de harmonia as relações do homem com os recursos naturais. O termo foi citado pela primeira vez, com essa acepção em 1972, no livro Blueprint for Survival (GOLDSMITH, ALLEN, ALLABY, 1972). Contudo, a segundo Van Bellen (2005) o entendimento de desenvolvimento sustentável surgiu no início dos anos de 1970, na crítica da sociedade ao modelo de desenvolvimento até então dominante, exclusivamente à base de crescimento econômico. De acordo com esse autor, o termo desenvolvimento sustentável foi debatido em dois eventos 
internacionais. O primeiro foi a Conferência de Estocolmo, de 1972, sobre meio ambiente humano, onde foi apreciado o relatório The limits to growth (MEADOWS et al., 1972), ressaltando-se que os problemas ambientais ocorriam numa escala global e aumentavam de forma exponencial. O segundo foi o Relatório da Comissão Bruntland, no qual desenvolvimento sustentável é descrito como capaz de satisfazer as necessidades presentes sem impedir que gerações futuras possam suprir as suas (CMMAD, 1988). Essa foi a primeira definição mundialmente aceita; a segunda, bem mais aceita atualmente, foi a do documento, de 40 capítulos, intitulado Agenda 21 (UNITED NATIONS, 1993).

Após a difusão desse documento, o termo sustentabilidade foi se tornando mundialmente conhecido, e cada vez mais utilizado em todos os setores da economia. No entanto, não há um consenso quanto ao conceito, pois o significado é distinto para diferentes pessoas e revela valores e percepções, na maioria das vezes, conflitantes sobre a utilização dos recursos naturais, e o desenvolvimento econômico e social. Desse modo, não há uma única definição de sustentabilidade, ou desenvolvimento sustentável, e nem agricultura sustentável. Porém, corroborando a construção da definição de sustentabilidade pelo viés da agricultura, destacam-se as ideias de autores clássicos e contemporâneos: Schultz (1965), Conway (1993), Tisdell (1999), Hammond et al., (1995) Sachs (2004) e Sepúlveda (2008).

Inicialmente, o clássico Schultz (1965), defende a premissa de que a adoção das novas tecnologias agrárias permite a elevação da renda de agroecossistemas intensivos, através do aumento da produção, da produtividade e do lucro, podendo gerar bons níveis de sustentabilidade nos agroecossistemas intensivos. Segundo esse autor situações como inundação de áreas férteis, modificação na qualidade do solo, alteração na qualidade da água, redução da mata nativa, processo erosivo, perda da biodiversidade etc. tenham originado problemas socioambientais avassaladores e com o emprego de técnicas de manejo adequado e gestão dos recursos naturais, dentre outras práticas, poderiam adequar-se à sustentabilidade.

No pensar do contemporâneo Conway (1993), a sustentabilidade deve ser percebida pela visão genérica, por isso mesmo adaptável às diversas atividades antrópicas, sem perder a referência da escala local ao longo do tempo. Esse autor define sustentabilidade como a habilidade de um sistema em manter sua produtividade quando está sujeito a intenso esforço ou a alterações. Isto é, sustentabilidade seria a capacidade de um sistema de manter ou melhorar sua produtividade de acordo com o potencial de seus recursos naturais e com a aptidão socioeconômica local.

como Tisdell (1999), reconhece-se aqui que sustentabilidade de sistemas agrícolas é mais um atributo a ser considerado no desempenho dos agroecossistemas, e que outros devem ser considerados, como produtividade e distribuição de renda. Além disso, no que diz respeito à sustentabilidade, deve-se esperar que a manutenção de determinado sistema agrícola tenha continuidade, não dependendo apenas dos fatores biofísicos, mas também dos sociais.

Por fim, Hammond et al. (1995), Sachs (2004) e Sepúlveda (2008) conceituam sustentabilidade como um processo multidimensional e intertemporal entre homem-natureza. Portanto a sustentabilidade deve ser praticada na escala local, tendo como eixos de ligação as dimensões ambiental (sustentabilidade), econômica (competitividade), social (equidade), dentre outras.

Refletindo às relações homem-natureza, não há apenas um limite mínimo para o bem-estar da sociedade humana, mas também um limite máximo para o uso e o manejo dos recursos naturais, de modo que os sistemas agrícolas sejam sustentáveis. Neste estudo, entendemos que praticar sustentabilidade nos agroecossistemas de bananeira, ou em unidades de análise, 
deve ser um processo que vise transformar a dinâmica do desenvolvimento in loco por meio de uma gestão sustentável de atividades produtivas, considerando o potencial dos recursos naturais e sociais centrados na escala local visando-se a que se sustente por longo período. Isto é, o uso dos recursos naturais deve ser elemento-chave de qualquer estratégia que objetiva o desenvolvimento sustentável, não só por sua importância para gerações futuras, mas por serem elementos cruciais para sustentação da vida.

\section{METODOLOGIA}

\subsection{CARACTERÍSTICAS DA METODOLOGIA}

A metodologia deste estudo caracteriza-se como pesquisa bibliográfica (GIL, 2009) e estudo de caso (YIN, 2007; SEPÚLVEDA, 2008), seguindo-se o método do desenvolvimento integrado sustentável (IDS) - biograma -, proposto por Sepúlveda (2008). Uma análise preliminar revelou que o método biograma poderia ser utilizado como ferramenta de caracterização e análise de sistemas agrícolas, permitindo, comparativamente, a avaliação dos níveis de sustentabilidade em diversas escalas de atividades antrópicas.

A seleção das unidades de análise obedeceu a dois critérios principais. Primeiro, elas unidades deveriam ser agroecossistemas intensivos de bananeira de alta tecnologia. Para isso, utilizaram-se dados secundários, do Instituto Brasileiro de Geografia e Estatística (IBGE) e da Federação da Indústria e Comércio do Rio Grande do Norte (FIERN) e dados primários (entrevista de campo 2011; 2012). Segundo, deveriam contemplar as dimensões e os indicadores ambientais, econômicos e sociais. Frente a isso, buscou-se em Gerardi e Silva (1981) - ao considerarem que a quantidade da amostra deve ser basicamente função do número de indivíduos componentes da população - a variabilidade e o nível de precisão desejados para as inferências da amostra.

Os agroecossistemas intensivos de bananeira pesquisados, de Ipanguaçu-RN foram os quatro deles que utilizam alta tecnologia. A amostra correspondeu ao percentual de $100 \%$ do universo. Após a escolha desses estabelecimentos,foram elaboradas as entrevistas estruturadas e o roteiro de observação não participante, com vista à coleta dos dados primários, para a análise quantitativa e qualitativa.

\subsection{TRATAMENTO DOS DADOS}

A metodologia para o tratamento dos dados partiu da definição de sustentabilidade, incorporando as seguintes características: sustentabilidade ${ }^{4}$, produtividade ${ }^{5}$, equidade ${ }^{6}$, em relação às dimensões ambiental, econômica e social, estruturadas por meio de quinze indicadores e respectivos parâmetros (Quadro 1). Foi excluída deste estudo a dimensão político-institucional, devido à dificuldade na obtenção de indicadores confiáveis e robustos para análise semelhantes aos aplicado para as outras dimensões, no período da pesquisa. A operacionalização dos dados deu-se no programa informatizado, planilha Microsoft Excel 2007, por meio do método de valores observados (SEPÚLVEDA, 2008), definindo-se, para todos os indicadores, os valores máximos e os mínimos.

Após a operacionalização, foram calculados os índices dos indicadores pelas médias aritmética e ponderada. Antes, porém, eles foram submetidos à função relação: positiva (+), quando o

\footnotetext{
${ }^{4}$ Habilidade do agroecossistema de manter a produtividade, a autorregulação e a resiliência ao ser submetido a stress (CONWAY, 1993; CÂNDIDO, 2010).

${ }^{5}$ Capacidade de agroecossistemas de produzir, em ton./ha., em determinado período (TRIOMPHE,1996).

${ }^{6}$ Aborda a justa distribuição da produtividade entre os agroecossistemas (SEPÚLVEDA, 2008).
} 
aumento no valor da variável resulta na melhoria do sistema agrícola; negativa (-), quando aumento no valor da variável resulta na piora do sistema agrícola. Para isso, realizou-se o procedimento de ajuste dos valores observados, conforme uma escala cujo valor mínimo era 0 (zero) e o máximo era 1 (um), tendo uma variação crescente de níveis, que simbolizava o estado de sustentabilidade: colapso, crítico, instável, estável e ótimo (Figura 1).

Figura 2 - Níveis do estado de sustentabilidade sob a ótica do biograma

\begin{tabular}{|c|c|c|c|c|}
\hline Colapso & & Instável & Estável & Ótimo \\
\hline Vermelho & Laranja & Amarelo & Azul & Verde \\
\hline 0 & & & & \\
\hline
\end{tabular}

Fonte: Adaptado de Sepúlveda (2008).

Em seguida, foram descritas as dimensões, com indicadores que foram estabelecidos para a avaliação de sustentabilidade dos agroecossistemas, bem como a função relação de cada indicador, considerando-se, ainda, parâmetros e fontes, conforme podem ser visualizados, também de maneira sistematizada, no Quadro 1.

A dimensão ambiental foi mensurada utilizando-se os seguintes indicadores: consumo de água - orçado por meio da quantidade de água usada diariamente pelo agroecossistema, considerando-se litro por planta, tendo como parâmetro a média de $\leq 30$ litro/planta, conforme Borges e Souza (2004) -; potencial hidrogeniônico $(\mathrm{pH})$ da água-medido conforme a concentração de íons hidrogênio $\mathrm{H}^{+}$, tendo como limites permissíveis valores que variam entre 6,0 e 9,5 conforme Apha et al. (2005) -; consumo de energia - calculado pelo consumo de energia em quilowatt-hora ao ano, tendo como parâmetro 0,36 Kwh/ha./a.a (pesquisa de campo 2012)-; potencial hidrogeniônico $(\mathrm{pH})$ do solo - avaliado conforme a concentração de íons hidrogênio $\mathrm{H}^{+}$, tendo como limites permissíveis valores entre 7,5 e 7,4, segundo Apha et al. (2005), Borges e Souza, (2004) -; área de reserva legal - contado conforme o percentual vegetação natural por hectare, tendo como limite legal o parâmetro $20 \%$ ha., mínimo da área total (BRASIL, 2012).

A dimensão econômica foi mensurada por meio dos seguintes indicadores: produção - orçada mediante volume produzido, em toneladas, no ano agrícola de 2011, tendo como parâmetro a produção do município de 29.475 toneladas (IBGE, 2011) -; produtividade - avaliada por meio da capacidade de produção do agroecossistema, toneladas por hectare (TRIOMPHE, 1996), tendo como parâmetro 37,5 ton./ha. (IBGE, 2010) -; confiança econômica - medida pela ponderação da importância do resultado líquido da produção, na situação, quando o preço dos produtos tem oscilação mais que $15 \%$ (COSTA, 2010), e quando o preço dos insumos sobe em igual proporção, conforme parâmetro de ponderação, 0 a 1 (pesquisa de campo, 2012) -; comercialização externa - mensurada pelo percentual comercializado de banana, no exterior, conforme exportação do município e parâmetro de 30.147 .849 kg (MDIC, 2011) -; empregabilidade permanente - quantificada do percentual de geração de emprego no agroecossistema com carteira assinada, em relação ao município, tendo como parâmetro 1.813 empregos (IBGE, 2010).

A dimensão social foi mensurada utilizando-se indicadores: escolaridade - calculada do percentual de trabalhadores rurais que com formação de educação básica, nos agroecossistemas, em relação ao número do município, conforme o parâmetro 90,4\% (IBGE, 2010; ODM, 2010) -; adequação de moradia própria - estimada pelo percentual de ocupação de domicílio próprio dos trabalhadores, em relação ao parâmetro municipal de 3.265 domicílios próprios (IBGE, 2010); uso de equipamento de proteção individual (EPIs) - avaliado o 
percentual de trabalhadores que fazem uso de EPIs, tendo-se como parâmetro a norma regulamentadora no 06, 2001 (MAPA, 2005); ocorrência de sinistro - quantificado o percentual, conforme parâmetro municipal (IBGE, 2010); acesso à justiça trabalhista calculado pelo percentual de acessos à justiça trabalhista dos trabalhadores dos agroecossistemas, conforme parâmetro municipal (IBGE, 2011; MT, 2010).

Quadro 1 - Dimensões, indicadores e parâmetros e fontes

\begin{tabular}{|c|c|c|c|}
\hline $\begin{array}{l}\text { Dimen } \\
\text { são }\end{array}$ & Indicador & $\begin{array}{l}\text { Rela } \\
\text { ção }\end{array}$ & Parâmetro e fonte \\
\hline \multirow{5}{*}{$\begin{array}{l}\text { Ambie } \\
\text { ntal* }\end{array}$} & A1-consumo de água & - & $\leq 30$ L/planta (BORGES; SOUZA, 2004) \\
\hline & A2 - pH da água de irrigação & - & 6,0-9,5 (APHA et al.2005) \\
\hline & A3 - consumo de energia & - & $\begin{array}{l}\text { 0,36Kwh/ha.(pesquisa de campo 2011; } \\
\text { 2012) }\end{array}$ \\
\hline & A4-pH do solo & - & $\begin{array}{l}\text { 7,5-7,4 (APHA et al.,2005; BORGES; } \\
\text { SOUZA, 2004) }\end{array}$ \\
\hline & A5 - área de reserva legal & + & 20\% (BRASIL, 2012) \\
\hline \multirow{5}{*}{$\begin{array}{l}\text { Econô } \\
\text { mica* }\end{array}$} & E1 - produção total & + & 29.475 ton.(IBGE, 2011) \\
\hline & E2 - produtividade & + & 37,5ton./ha.(TRIOMPHE,1996; IBGE, 2011) \\
\hline & E3 - confiança econômica & + & 0 a 1 (pesquisa de campo 2011) \\
\hline & E4 - comercialização & + & 30.147.849 kg (IBGE, 2011) \\
\hline & E5 - empregabilidade & + & 1.813 (IBGE, 2010) \\
\hline \multirow{5}{*}{$\begin{array}{l}\text { Social } \\
* * *\end{array}$} & S1-escolaridade & + & 90,4\% (IBGE, 2010; PORTAL ODM, 2011) \\
\hline & S2 - adequação moradia & + & 3.265 (IBGE, 2010); \\
\hline & $\begin{array}{l}\text { S3 - equipamento de } \\
\text { proteção individual }\end{array}$ & + & NR no 06, 2001 (MAPA, 2005) \\
\hline & S4 - ocorrência de sinistro & - & 0 a 1 (SESP,2011; pesquisa de campo) \\
\hline & $\begin{array}{l}\text { S5 - acesso à justiça } \\
\text { trabalhista }\end{array}$ & + & 149 (MT, 2011) \\
\hline
\end{tabular}

Fonte: Pesquisa de campo, 2012.

*Dimensão de avaliação ambiental - indicadores $\mathrm{A} ;{ }^{* *}$ Dimensão de avaliação econômica - indicadores $\mathrm{E} ; \mathrm{e}^{* * *}$ Dimensão de avaliação social - indicadores $\mathrm{S}$.

\subsection{CARACTERIZAÇÃO DOS AGROECOSSISTEMAS DE PRODUÇÃO DE BANANA}

Este estudo de caso foi realizado em quatro agroecossistemas intensivos de bananeira que utilizam alta tecnologia, os quais foram escolhidos por apresentarem diferentes características na produtividade, na média de consumo de água de irrigação e na empregabilidade.

Esses quatro AGROs, localizados no município de Ipanguaçu, no estado do Rio Grande do Norte, estão historicamente vinculados a certa relevância, por fazerem parte do Vale do Açu, o qual tem sido de grande importância socioeconômica e ambiental para a região, já que é considerado como o vale fluvial de maior volume de água superficial local, possibilitando a implantação de projetos de irrigação de fruticultura no município de Ipanguaçu-RN (Figura 1).

O município de Ipanguaçu tem uma área territorial de $374,236 \mathrm{Km}^{2}$, clima semiárido, precipitação pluviométrica anual observada de $662.1 \mathrm{~mm}$, período chuvoso entre fevereiro e maio, e temperaturas médias de $27,9{ }^{\circ} \mathrm{C}$ (IDEMA, 2008). A população residente é de aproximadamente 13.856 habitantes - assim distribuída: 5.383 na área urbana e 8.473 na área rural - resultando numa densidade demográfica de 37,02 hab./Km² (IBGE, 2011).

Os agroecossistemas que utilizam alta tecnologia avaliados neste estudo correspondem aos existentes à margem direita do Rio Piranhas Açu - de onde captam água para irrigação -, na 
planície fluvial, com altitude média de $23,7 \mathrm{~m}$ e formada por solos aluviais. Abrangem mais de 786 ha. de área plantada e têm rendimento médio de 37,5 ton./ ha. de banana (IBGE, 2011).

Na Tabela 2, são apresentadas a produção de bananas nacional, a regional, a estadual, a microrregional, a municipal e a dos agroecossistemas, no ano agrícola de 2011 , bem como o percentual da produção dos agroecossistemas em relação às escalas mencionadas referentes à produção de banana, em toneladas, no ano de 2011 (IBGE, 2011; pesquisa de campo, 2012).

Tabela 2 - Produção de banana, 2011

\begin{tabular}{|l|r|r|}
\hline Escalas & Toneladas (ton.) & $\%$ \\
\hline Brasil & 7.329 .471 & 0,46 \\
\hline Nordeste brasileiro & 2.862 .505 & 1,19 \\
\hline Rio Grande do Norte & 142.750 & 23,87 \\
\hline Microrregião Vale do Açu-RN & 76.523 & 44,52 \\
\hline Município de Ipanguaçu-RN & 29.475 & 115,6 \\
\hline Agroecossistemas intensivos & $34.074 *$ & 100 \\
\hline
\end{tabular}

Fonte: IBGE (2011), MDIC (2011).

*Pesquisa de campo (2011).

$\mathrm{Na}$ tabela, 2 destaca-se a elevada participação produtiva dos quatro agroecossistemas intensivos de bananeira (pesquisa de campo, 2011) quanto à produção agrícola municipal (IBGE,2011), em relação à escala nacional $(0,46 \%)$, à regional $(1,19 \%)$, à estadual $(23,87 \%)$, à microrregional $(45,85 \%)$ e à municipal $(115,6 \%)$. Os dados percentuais revelam elevada produção de banana nos AGROs, em relação ao município, à microrregião e ao estado. Porém, tal característica, apesar de contribuir para a geração de empregos local, promove diferença quantitativas no tocante à distribuição do número de empregos por gênero, predominando os homens, isto é, a distribuição de emprego não é equitativa (Tabela 3).

Tabela 3 - Distribuição de empregos por agroecossistema e gênero

\begin{tabular}{l|c|c|c|c}
\hline Gênero & AGRO 1 & AGRO 2 & AGRO 3 & AGRO 4 \\
\hline Mulheres & 12 & 14 & 41 & 15 \\
Homens & 160 & 138 & 269 & 165 \\
\cline { 2 - 5 } Soma & 172 & 152 & 310 & 180 \\
\hline
\end{tabular}

Fonte: Pesquisa de campo (2011).

A Tabela 3 expõe a quantidade reduzida de empregos disponíveis para mulheres em relação aos disponíveis para os homens. Apesar das peculiaridades na distinção entre trabalho de homem e trabalho de mulher e das exigências de redução do trabalho manual em momentos de ocorrência das intempéries climáticas ou nas crises econômicas da fruticultura, observa-se, efetivamente, um número de 82 mulheres, que estão desenvolvendo suas atividades no setor administrativo, na secretaria, na recepção, na linha de produção empacotadeira, no serviço geral, dentre outros setores (pesquisa campo, 2011).

A principal característica desses AGOs é a produção de banana centrada em princípios da maximização da produtividade, da comercialização e do lucro (VEIGA, 2006; BORLAUG, 1997; 2008). A média de produtividade de 46 ton./ha., é superior ao rendimento médio obtido no município, 37,5 ton./ha. (IBGE, 2011). Setenta e cinco por cento são destinados a comercialização ao mercado externo, $8 \%$ são destinado ao mercado interno, isto é, às indústrias de beneficiamento - de doces e de bananas secas - e $17 \%$ são destinados à comercialização in natura (Pesquisa de campo, 2012). 
Os agroecossistemas de alta tecnologia, multinacionais, estão distribuídos espacialmente nas seguintes comunidades: Baldum - no sudoeste municipal, está o $A G R O 1$, distando 6,6 km da sede administrativa municipal, entre a latitude $5^{\circ} 4^{\prime} 01.07^{\prime \prime} \mathrm{S}$ e a longitude $36^{\circ} 52^{\prime} 30.93^{\prime \prime} \mathrm{W}$.; principais características: dimensões de 113.11, produtividade de 43,93 ton./ha., empregabilidade:172 pessoas (Pesquisa de campo, 2012). Base Física - no oeste municipal, está o $A G R O 2$, distando $4,6 \mathrm{~km}$ da sede administrativa municipal, entre a latitude $5^{\circ} 32^{\prime} 32.90^{\prime \prime}$ S e a longitude $36^{\circ} 52^{\prime} 43.38^{\prime \prime} \mathrm{W}$; principais características: dimensão 150,92 ha., produtividade 37,92ton./ha., empregabilidade: 152 pessoas (Pesquisa de campo, 2012). Olho D'água - no centro municipal, está o $A G R O 3$, distando $1 \mathrm{~km}$ da sede administrativa municipal, entre a latitude $5^{\circ} 31^{\prime} 14.39^{\prime \prime} \mathrm{S}$ e a longitude $36^{\circ} 51^{\prime} 88.00^{\prime \prime} \mathrm{W}$, principais características: dimensão de 274.39 ha., 58,45 ton./ha., empregabilidade: 310 pessoas (Pesquisa de campo, 2012). Baldum - no sudoeste municipal, está o $A G R O 4$, distando $5,6 \mathrm{~km}$ da sede administrativa municipal, entre a latitude $5^{\circ} 33^{\prime} 27^{\prime \prime} \mathrm{S}$ e a longitude $36^{\circ} 52^{\prime} 28653^{\prime \prime} \mathrm{W}$; principais características: dimensões de 173.56 ha., produtividade de 42,32 ton./ha., empregabilidade: 180 pessoas (Pesquisa de campo, 2012).

\section{RESULTADOS E DISCUSSÃO}

Os índices de sustentabilidade são números que permitem a comparação entre diferentes indicadores, dentro deles e entre diferentes dimensões (Tabela 4). Dessa forma, foi possível uma análise comparativa entre índices, compostos por uma parte de dados ambientais, outra parte econômica e uma terceira parte social. Como resultado, tem-se que os agroecossistemas 2 e 4 apresentaram índices de 0,33 e 0,24, respectivamente, indicando um estado crítico de sustentabilidade; o agroecossistema 1 apresentou o índice de 0,47, apontando estado instável, e o agroecossitema 3 apresentou o índice de 0,94, demonstrando um estado ótimo. Concluise, portanto, que esse último agroecossistema é o mais sustentável. Entretanto, mesmo tendo nível de sustentabilidade ótimo, o AGRO 3, no indicador fertilidade do solo, obteve baixo o mais baixo índice $(0,06)$, em relação aos agroecossistemas 2 e 4 . Na Tabela 4, sistematizamos as dimensões, os indicadores, e os índices de sustentabilidade dos agroecossistemas.

Tabela 4 - Dimensões, indicadores e índices de sustentabilidade dos agroecossistemas

\begin{tabular}{llllll}
\hline \multicolumn{1}{c}{ Indicador } & AGRO 1 & AGRO 2 & AGRO 3 & AGRO 4 \\
\hline $\begin{array}{lllll}\text { Ambiental } \\
\text { (A) }\end{array}$ & A1 - consumo de água & 0,43 & 0,62 & 1 & 0 \\
& A2 - pH da água & 0,34 & 0 & 1 & 0 \\
& A3 - consumo de energia & 0 & 0,27 & 1 & 0,57 \\
& A4 - pH do solo & 0 & 0,56 & 0,06 & 1 \\
& A5 - área de reserva legal & 1 & 0 & 1 & 1 \\
\hline \multirow{5}{*}{ Econômica } & E1 - produção & 0 & 0,07 & 1 & 0,21 \\
(E) & E2 - produtividade & 0,29 & 0 & 1 & 0,2 \\
& E3 - confiança econômica & 1 & 0,50 & 1 & 0,0 \\
\hline Social & E4 - comercialização & 0 & 0,07 & 1 & 0,21 \\
(S) & E5 - empregabilidade & 0,13 & 0 & 1 & 0,18 \\
& S1 - escolaridade & 0,69 & 0 & 1 & 0,44 \\
& S2 - adequação moradia & 0,13 & 0 & 1 & 0,18 \\
& S3 - uso de equipamentos de & 1 & 0 & 1 & 1 \\
& proteção individual & & & & \\
& S4 - ocorrência de sinistro & 1 & 0,5 & 1 & 0 \\
& S5 - acesso à justiça trabalhista & 1 & 1 & 1 & 0 \\
\hline
\end{tabular}

Fonte: Elaboração dos autores (2012). 
Nos biogramas 1, 2, 3 e 4, está exposto o estado de sustentabilidade dos agroecossistemas intensivos (AGROs) no ano agrícola de 2011. Observa-se um nítido desequilíbrio dos AGRO1, AGRO2 e AGRO4 em relação ao AGRO3, revelado nas dimensões ambiental, econômica e social.

A seguir, os biogramas 1, 2, 3, e 4, mostram com mais consistência a contribuição de cada indicador de sustentabilidade dos agroecossistemas, até mesmo quais indicadores de desenvolvimento da cultura de banana devem ser objeto de políticas públicas. Quanto maior e mais homogênea área colorida, ou sombreada, maior o desempenho sustentável do agroecossitema.

Biograma 1- AGRO 1

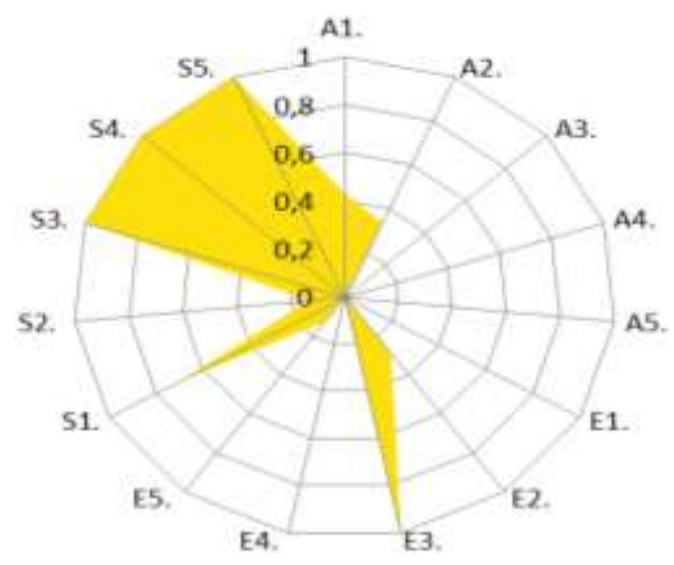

Biograma 3 - AGRO 3

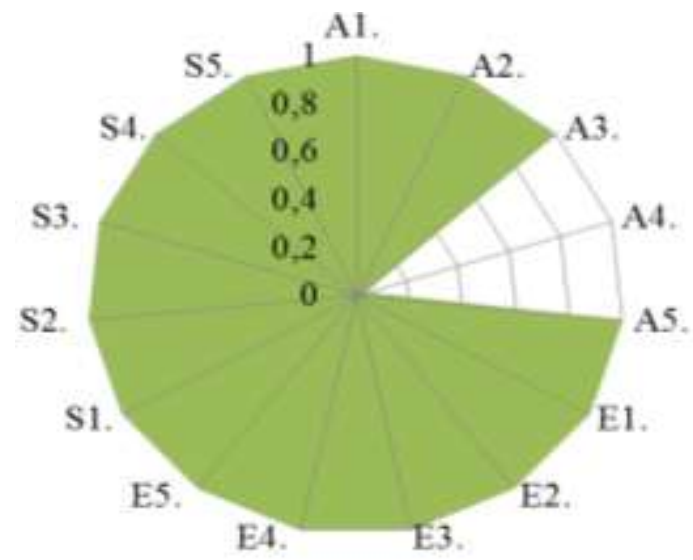

Biograma 2 - AGRO 2

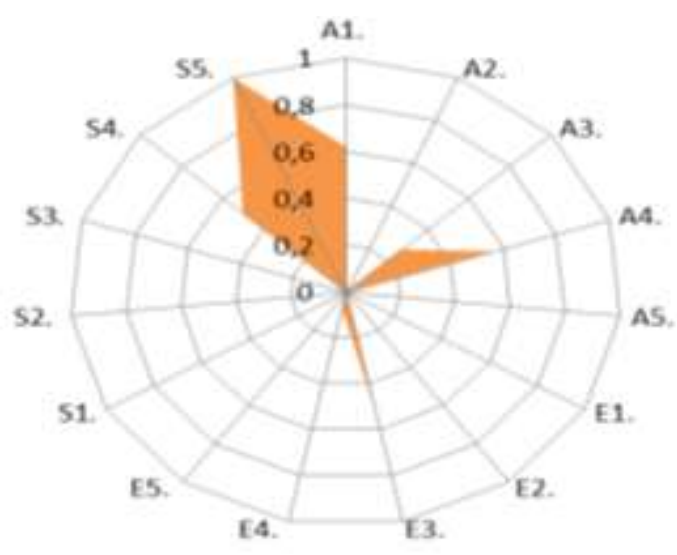

Biograma 4 - AGRO 4

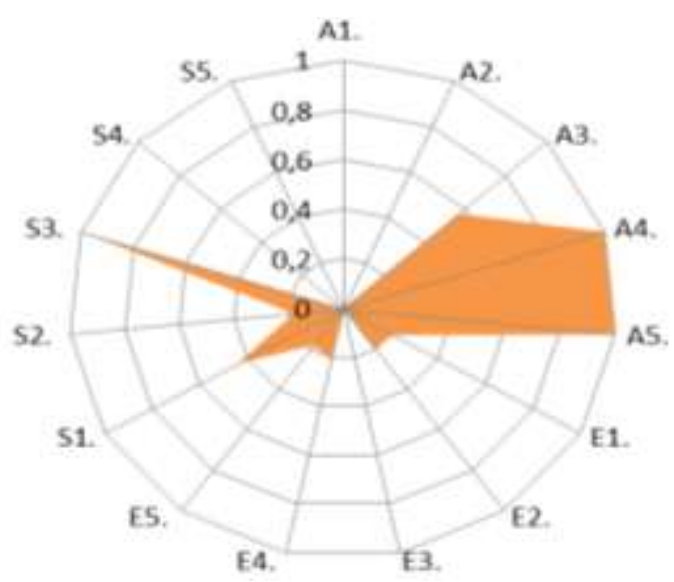

Fonte: Elaboração dos autores (2012).

Após a visualização individual dos quatro biogramas dos agroecossistemas, acima, e seguindo a lógica comparativa, percebe-se que a maior área sombreada corresponde ao AGRO3 e a menor ao AGRO2. A seguir, são apresentados os índices de cada dimensão, com o objetivo de se estabelecer uma comparação também entre as dimensões de um mesmo AGRO, possibilitando comparar situações de desarmonia ou harmonia em relação ao desenvolvimento sustentável.

O biograma 1 expõe a situação do AGRO 1, um estado instável de equilíbrio, em relação às demais unidades de análise, conforme a Tabela 4. Entretanto, na dimensão social, os indicadores S3, S4 e S5 estão no nível ótimo, quando essa dimensão é comparada às demais, excetuando-se à dimensão econômica, em que o indicador E3 apresentou índice ótimo. O AGRO 1 apresentou índice de sustentabilidade 0,47 , estado instável. 
O biograma 2 expõe a situação do AGRO 2, um estado instável de equilíbrio, em relação às demais unidades de análise, conforme Tabela 4. Entretanto, na dimensão social, o indicador S5 está no nível ótimo, quando essa dimensão é comparada às demais. O AGRO 2 apresentou índice de sustentabilidade 0,24 , estado crítico.

O biograma 3 expõe a situação do AGRO 3, um estado ótimo de equilíbrio, em relação às demais unidades de análise, conforme dados da tabela 4. Entretanto, na dimensão ambiental, o indicador A4 está no nível colapso quando essa dimensão comparada às demais. Portanto, o AGRO3 apresentou índice de sustentabilidade 0,94, estado ótimo;

O biograma 4 expõe a situação do AGRO 4, um estado instável de equilíbrio, em relação às demais unidades de análise, conforme dados da Tabela 4. Entretanto, na dimensão ambiental, os indicadores A4 e A5, assim como na dimensão social o indicador S3, estão no nível ótimo, quando essa dimensão é comparada às demais. O AGRO 4 apresentou índice de sustentabilidade 0,33, estado crítico.

Por fim, no biograma 5 sintetizam-se os índices dos quatro agroecossistemas intensivos, para visualização, e sobrepõem-se os distintos estados de sustentabilidade dos sistemas agrícolas. Faz-se importante destacar que o biograma é a imagem, mas não expressa as trajetórias traçadas, ou seja, a dinâmica das unidades de análises, evolutivamente. Este estudo corresponde temporalmente ao ano agrícola de 2011.

\section{Biograma 5 - Agroecossistemas 1, 2,3 e 4}

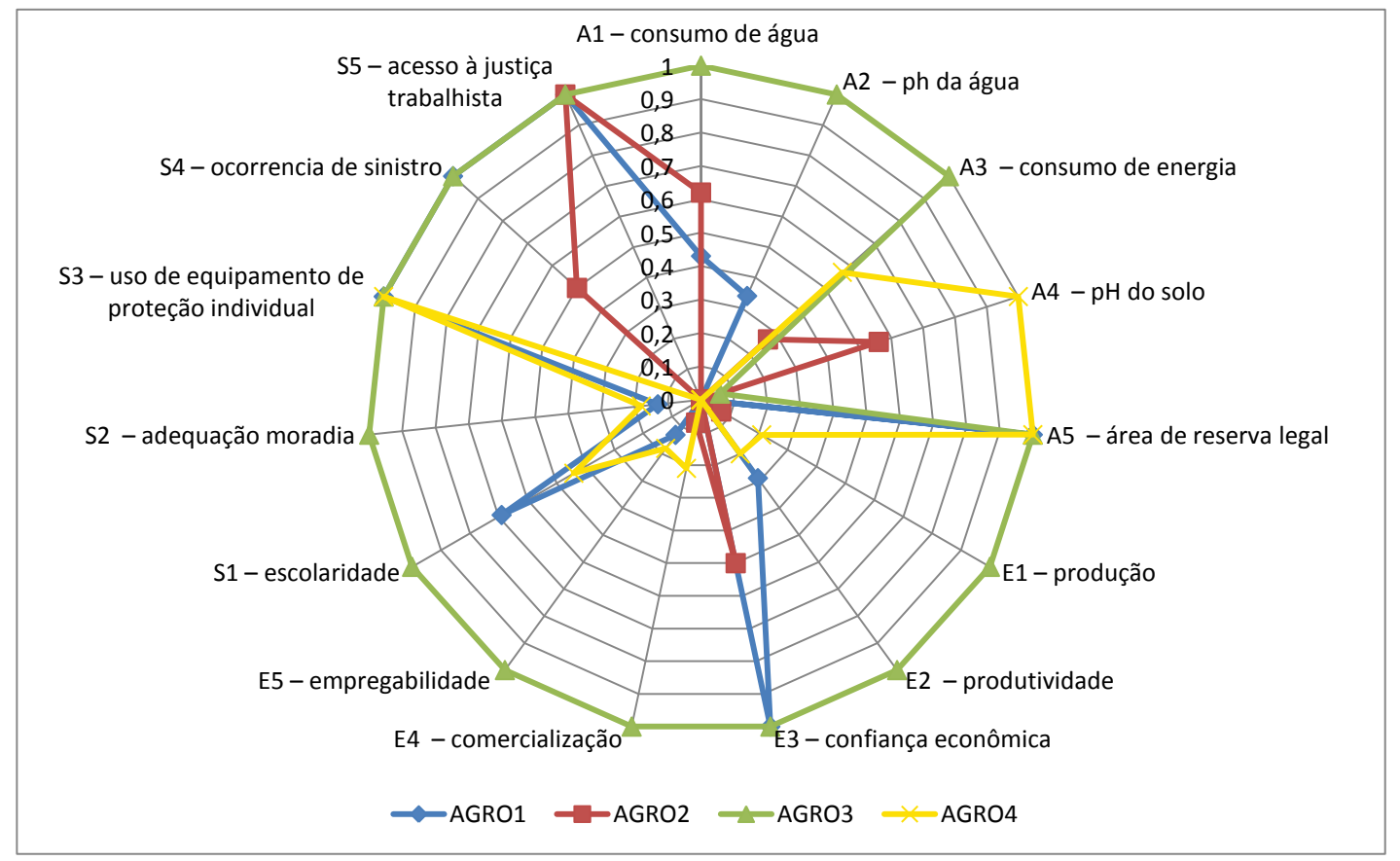

Fonte: Elaboração dos autores (2012).

A aplicação do método biograma (SEPÚLVEDA, 2008), neste estudo, permitiu a compreensão dos processos de desenvolvimento, dos aparentes desequilíbrios dimensionais dos agroecossistemas intensivos. Quanto ao indicador produtividade, por exemplo, os agroecossistemas apresentaram os seguintes resultados: AGRO1 - 43,93 ton./ha.; AGRO2 - 37,92 ton./ha.; AGRO3 - 58,45 ton./ha.; AGRO4 - 42,32 ton./ha. Eles superaram o parâmetro local (37,5 ton./ha.,) respectivamente, em $17,15 \%$ ton./ha.; $1,12 \%$ ton./ha.; $55,86 \%$ ton./ha.; $12,85 \%$ ton./ha. de banana (pesquisa de campo 2012). Dentre eles, o AGRO 2 foi o que teve o mais baixo desempenho.

\section{CONSIDERAÇÕES FINAIS}


Diante dos resultados dos índices de sustentabilidade dos indicadores, mesmo estando distante um consenso sobre sustentabilidade de agroecossistemas intensivos, considera-se a importância de se trabalhar com os conceitos de sustentabilidade, produtividade e resiliência nos sistemas de cultivo de bananeira locais.

Quanto à produtividade, o nível de sustentabilidade dos agroecossitemas configura um estado ótimo. $\mathrm{E}$, quanto à sustentabilidade dos agroecossistemas intensivos no que diz respeito aos recursos naturais - água, solo e vegetação nativa - considera-se de uma maneira geral, que esses agroecossitemas demonstram nível de sustentabilidade um estado instável.

No entanto, quanto à equidade, no que diz respeito à justa distribuição social e da produtividade entre os agroecossistemas, configura-se um nível de sustentabilidade em estado colapso.

Ademais, o AGRO3 aparece como o mais sustentável, estado ótimo, em comparação aos demais. Em contrapartida o AGRO2 mostra ser o menos sustentável, em estado de colapso. A interpretação das imagens projeta a necessidade de diferentes formas de intervenção na gestão de manejo de produção da banana e dos recursos naturais, bem como da adoção de práticas socioeconômicas que assegurem a sustentabilidade local ao longo do tempo.

Por fim, percebe-se a necessidade de aprofundar metodologias de avaliação da sustentabilidade que contemplem os agroecossistemas intensivos - entendendo-se a importância do esclarecimento de questões como sustentabilidade, equidade e produtividade local em longo prazo, por serem esses sistemas agrícolas também difusores da segurança alimentar e nutricional - além de desenvolver tecnologias e práticas no manejo que possam promover caminhos sustentáveis para fruticultura de Ipanguaçu-RN.

Este artigo é parte da tese de doutoramento do primeiro autor, que agradece ao Conselho Nacional de Desenvolvimento Científico e Tecnológico (CNPq) o apoio financeiro.

\section{REFERÊNCIAS}

ALBANO, G. P. Globalização da agricultura e concentração fundiária no município de Ipanguaçu-RN. Recife: UFPE, 2008.

ALTIERI M. Biotecnologia agrícola. Porto Alegre: ASCAR-EMATER/RS, 2002.

ANDRADE, M.C. de. A questão do território no Brasil. São Paulo: Hucitec, 1995.

ANTAS, F. P. de S. Avaliação da composição iônica e aplicação de um índice de qualidade para água de irrigação no rio Açu, RN. 2011. 68 f. Dissertação (Mestrado Irrigação e Drenagem)-Universidade Federal Rural do Semiárido, Mossoró, 2011.

APHA et al. Standard methods for the examination of water and waste-water, 21 th. Washington, DC: American public health Associations, 2005.

BORGES, A.L.; SOUZA, L. S. O cultivo da bananeira. Cruz das Almas: Embrapa mandioca e fruticultura, 2004.

BORLAUG, N. E. Factual errors and misinformation: Norman Borlaug defends the green revolution. Ecologist, v. 27, n. 5, p. 211, 1997.

BORLAUG, N.E. Agriculture's global hero continues to inspire. In: .Confronting crisis: agriculture and global development in the next fifty years. Des Moines, lowa: 2008. 
BRASIL. Medida provisória nํㅜㄹ.166-67, de 24 de agosto de 2001. Disponível em:< http://www.planalto.gov.br/ccivil 03/ Ato2011-2014/2012/Lei/L12651.htm\#art83>. Acesso em: 10 maio 2012.

.Lei federal $n^{\circ}$ 9.985/2000. Disponível em: <www.planalto.gov.br/ccivil 03/leis/L9985.htm>. Acesso em: 11 jan.2012.

BRASIL. Lei Federal no 12.727/12. Código florestal brasileiro. Medida provisória 571/12. Disponível em:<ttp://www.in.gov.br/visualiza/index.jsp?data=28/05/2012\&jornal>. Acesso 29 Out. 2012.

COELHO, E. F. Curso de bananicultura irrigada. Cruz das Almas: Embrapa, 2009.

COMISSÃO MUNDIAL SOBRE O MEIO AMBIENTE E DESENVOLVIMENTO. Nosso futuro comum. Relatório Brundtland. Rio de Janeiro: Fundação Getúlio Vargas, 1988.

CÂNDIDO, G. A. Desenvolvimento sustentável e sistemas de indicadores de sustentabilidade. Campina Grande: Ed. UFCG, 2010.

CONWAY, G.R. Análise participativa para o desenvolvimento agrícola sustentável. Rio de Janeiro: AS-PTA, 1993.

DINIZ, J. A. F. Geografia da agricultura. São Paulo: Difel, 1986.

FEDERAÇÃO DAS INDÚSTRIAS DO ESTADO DO RIO GRANDE DO NORTE. Disponível em: <http://www.fiern.org.br>. Acesso em: 10 mar. 2011.

GERARDI, L.H; SILVA, B.C.N. Quantificação em Geografia. São Paulo: DIFEL, 1981.

GIL, A. C. Como elaborar projetos de pesquisa. São Paulo: Atlas, 2009.

GOLDSMITH, E, ALLEN, R., ALLABY, M. A blueprint for survival. Boston: Penguin, 1972

GOMES, A. S. O emprego parcial na agricultura: a pluriatividade no polo fruticultor do Rio Grande do Norte. São Paulo: FEA/USP, 1999.

HAMMOND, A., et al. Environmental indicators: a systematic approach to measuring and reporting on environmental policy performance in the context of sustainable development. Washington DC: World Resources Institute, 1995.

INSTITUTO BRASILEIRO DE GEOGRAFIA E ESTATÍSTICA Produção agrícola municipal 2011. Disponível em:< http://www.sidra.ibge.gov.br/bda/agric/default.asp?z=t\&o=11\&i=P>. Acesso em: 29 out. 2012.

MEADOWS, D.H., MEADOWS, D.L., RANDERS, J., BEHRENS III, W.W. The limits to growth: a report for the club of Romés project on the predicament of Mankind. New York: Universe books, 1972.

MINISTÉRIO DO DESENVOLVIMENTO, INDÚSTRIA E COMÉRCIO EXTERIOR. Exportação municípios. Brasília: MDIC, 2011. Disponível em: http://www.desenvolvimento.gov.br. Acesso em: 10 jan. 2011.

MINISTÉRIO DA AGRICULTURA, PECUÁRIA E ABASTECIMENTO. Instrução normativa no 001, de $\mathbf{2 0}$ de janeiro de 2005. Disponível em:< http://www.inmetro.gov.br>. Acessado em: 20 jun. 2012.

PORTAL ODM. Relatório dinâmico RN Ipanguaçu, 2011. Disponível em: < https://www.portalodm.com.br/relatorios/PDF/gera_PDF ipanguaçu >. Acesso em: 14 maio 2011.

RIO GRANDE DO NORTE. Perfil do seu município: Ipanguaçu. Natal: IDEMA, 2008. 
SACHS, I. Desenvolvimento: includente, sustentável, sustentado. R J: Garamond, 2004.

SAMPAIO, Y. Programas e projetos de apoio ao desenvolvimento da agricultura irrigada: polos e culturas principais, destino da produção. Recife: PIMES, 2003.

SANTOS, M.; SILVEIRA, M.L. O Brasil: território e sociedade no início do século XXI. Rio de Janeiro: Record, 2002.

SCHULTZ, T. W. A transformação da agricultura tradicional. Rio de Janeiro: Zahar, 1965.

SECRETARIA DE ESTADO DA SAÚDE PÚBLICA. Estatística dos agravos relacionados ao trabalho acidente de trabalho. Natal, RN: SINAN, 2011.

SEPÚLVEDA, S. S. Biograma: metodología para estimar el nivel de desarrollo sostenible de territorios. San José, C.R.: IICA, 2008.

SILVA, A. F. Município de Ipanguaçu-RN. Natal: IFRN-NESA, 2012. 1 mapa. Escala 1: 160.000.

TISDELL, C. Biodiversity, conservation and sustainable development: principles and practices with Asian examples. Reino Unido e Estados Unidos: Edward Elgar, 1999.

TRIOMPHE, B. Rendimiento de maíz en milpas de campesinos. Red Gestión de Recursos Naturales, v. 5, p. 22-31, 1996.

UNITED NATIONS. Report of the united nations conference on environment and development. Rio de Janeiro: UN, 1993.

VALVERDE, O.; MESQUITA, M. Geografia Agrária do Baixo Açu. Revista Brasileira de Geografia, v. 23, n. 3, p. 455-494.

VAN BELLEN, H. M. Indicadores de sustentabilidade: uma análise comparativa. Rio de Janeiro: Editora FGV, 256p. 2005.

VEIGA, J. E. Desenvolvimento sustentável: o desafio do século XXI. Rio de Janeiro: Garamond, 2006.

VIETMEYER, N. Harmonizing biodiversity conservation and agricultural development. In: SRIVASTAVA J. P,;SMITH, N.H. FORNO, D. A. Biodiversity and Agricultural Intensification. Partners for Development and Conservation. Washington, DC: The World Bank, 1996. p. 11-30.

. Norman Borlaug. Boletim da federação brasileira de plantio direto na palha, ano 10, n. 37. p. 7. Jul./Set. 2009.

YIN, Robert K. Estudo de caso: planejamento e métodos. São Paulo: Bookman, 2007. 